\title{
Rapid calculation of a Centroid Moment Tensor and waveheight predictions around the north Pacific for the 2011 off the Pacific coast of Tohoku Earthquake
}

\author{
Jascha Polet $^{1}$ and Hong Kie Thio ${ }^{2}$ \\ ${ }^{1}$ Department of Geological Sciences, California State Polytechnic University, Pomona, CA 91768 \\ ${ }^{2}$ URS Corporation, 915 Wilshire Blvd, suite 700, Los Angeles, CA 90017
}

(Received April 5, 2011; Revised May 2, 2011; Accepted May 7, 2011; Online published September 27, 2011)

\begin{abstract}
We present the results of a near real-time determination of a Centroid Moment Tensor for the 2011 Tohoku quake and the subsequent rapid prediction of Pacific coast tsunami waveheights based on these CMT parameters. Initial manual CMT results for this event were obtained within 23 minutes of origin time and fully automatic results were distributed by E-mail within 33 minutes. The mechanism, depth and moment magnitude were all well constrained, as was indicated by a bootstrapping analysis. Using an existing library of tsunami Green's functions, we computed predicted waveheights in the north Pacific for several scenarios of the Tohoku earthquake that are consistent with the CMT solution. Overall, these predicted waveheights correspond well with preliminary observations around the Pacific Rim. The predictions for North America were sent out three and a half hours after the origin time of the earthquake, but this system has the potential to provide these predictions within minutes after receiving the CMT solution.
\end{abstract}

Key words: Real-time earthquake source analysis, tsunami early warning, 2011 Tohoku earthquake, Centroid Moment Tensor.

\section{1. rCMT}

The rCMT (research CMT) system computes fully automated Centroid Moment Tensors (CMTs) for large worldwide earthquakes using long period surface waves and is currently operational at the National Earthquake Information Center (NEIC) of the United States Geological Survey (USGS) in research/evaluation mode. One of the missions of the USGS NEIC is to rapidly determine the location and size of all destructive earthquakes worldwide and to immediately disseminate this information to concerned national and international agencies, scientists, and the general public. The rCMT system's main purpose is to calculate very rapid reliable moment magnitude estimates and mechanisms for earthquakes greater than 7.0 without the requirement of a human operator, in order to help assess the appropriate level of NEIC response after a large global earthquake, both in terms of the needed response staff and the level of urgency in generating and reviewing derived data products such as PAGER (Earle et al., 2009) and ShakeMap (Wald et al., 2003) that provide impact estimates. The method used to compute the CMTs is based on Dziewonski et al. (1981), as also used by the Global CMT group, but input waveforms are filtered to 130 to $330 \mathrm{~s}$. We calculate excitation kernels for 6 independent components of the moment tensors generated by summation of normal modes. A synthetic seismogram is a linear combination of these six

Copyright (C) The Society of Geomagnetism and Earth, Planetary and Space Sciences (SGEPSS); The Seismological Society of Japan; The Volcanological Society of Japan; The Geodetic Society of Japan; The Japanese Society for Planetary Sciences; TERRAPUB.

doi:10.5047/eps.2011.05.005 traces and the goal of the inversion is to find the weights that give the best agreement between the observed and synthetic seismograms. A least squares condition leads to an initial estimate of the moment tensor. In the full CMT inversion, the initial, hypocentral, parameters (location/origin time) are then perturbed in subsequent iterations. More information about the basic inversion methodology may be found in Kawakatsu (1989) and Polet and Kanamori (1995). An Email list is used to distribute these rCMT solutions, (http:// geohazards.cr.usgs.gov/mailman/listinfo/researchcmt).

Advantages to using these long period waveforms include:

- The effect of 3-D heterogeneity is minimized, so 1-D mode synthetics, involving only relatively few normal modes, can be used. The synthetics can thus be computed very quickly.

- The directivity effects are limited for events with magnitude $<8.5$, so a point source approximation is appropriate.

- Large aftershocks can still be analyzed, in cases where body wave techniques suffer from interference effects from the mainshock surface waves.

Disadvantages to using these long period waveforms include:

- Surface waves need to travel some distance before they are fully developed and close-in stations may be clipped, so we do not use stations at distances less than $10^{\circ}$ from the earthquake.

- Long period surface wave inversions for very shal- 


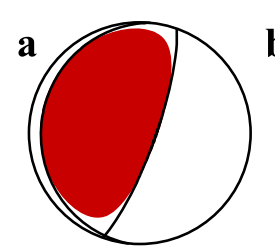

$186 / 12 / 76$

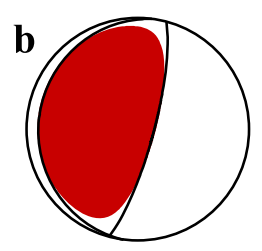

$187 / 12 / 83$

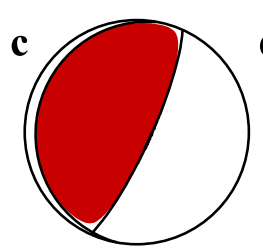

$190 / 11 / 76$

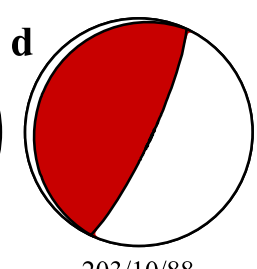

$203 / 10 / 88$

Fig. 1. Centroid moment solutions for the Tohoku earthquake, from left, (a), to right, (d): manually determined rCMT solution 23 minutes after origin time (depth $24 \mathrm{~km}, M_{\mathrm{w}} 8.9$ ); fully automatic rCMT sent out 33 minutes after origin time (depth $24 \mathrm{~km}, M_{\mathrm{w}} 8.9$ ); final rCMT determined 12 hours after origin time (using all available waveform data; depth $20 \mathrm{~km}, M_{\mathrm{w}}$ 9.0); gCMT solution (depth $20 \mathrm{~km}, M_{\mathrm{w}}$ 9.1). Also shown are the strike, dip and slip of the plane of the best fitting double couple that corresponds to the fault plane.
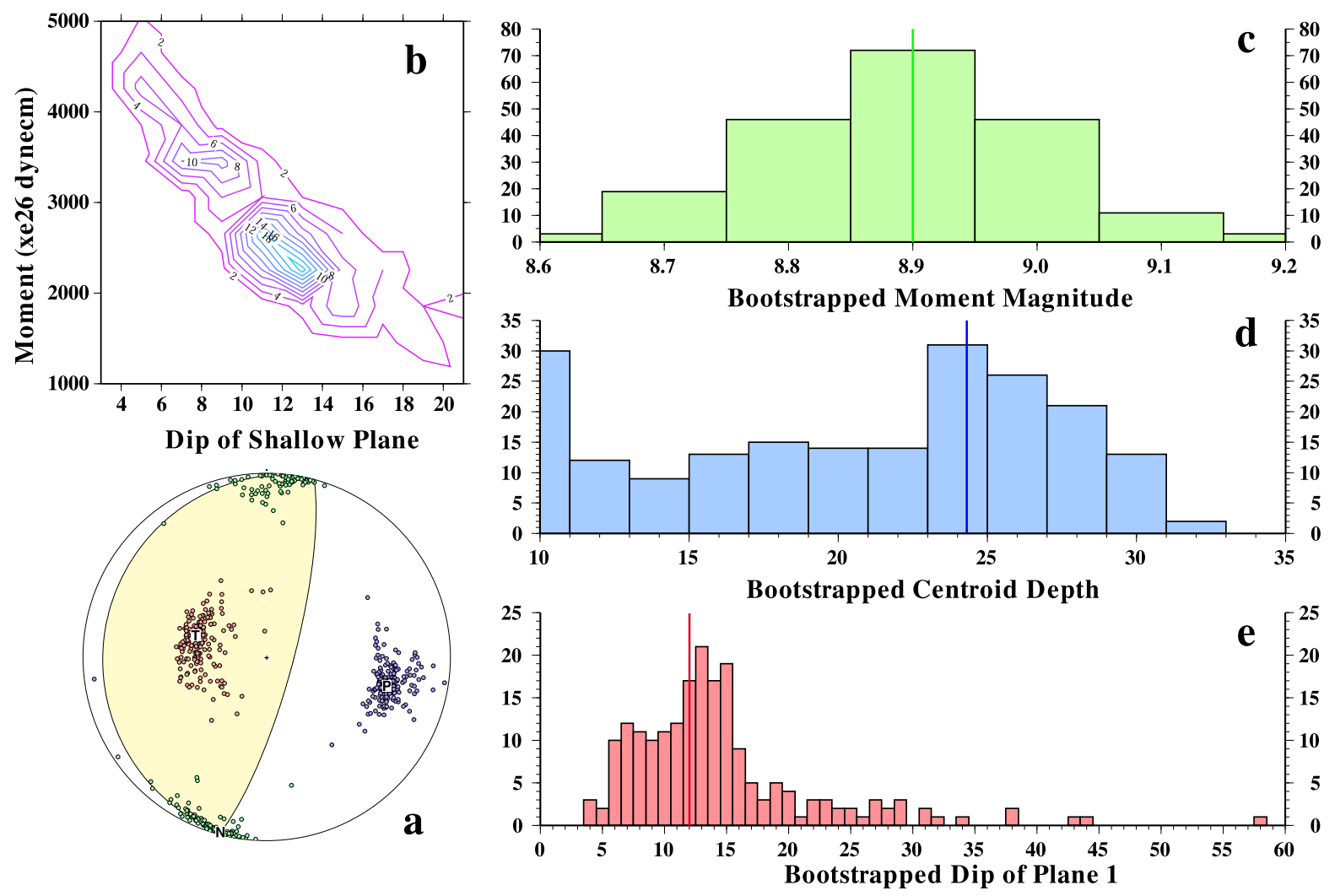

Fig. 2. Results of bootstrapping analysis of fully automatic rCMT solution, obtained 11 minutes after this solution was sent out. rCMT solution parameters are indicated by solid lines in histograms for moment magnitude, centroid depth and dip of the shallowly dipping plane, (right panels, c, $\mathrm{d}$ and e) and fault planes in the mechanism (bottom left panel, a). Axes shown in focal mechanism plot as well as moment magnitude, depth and dip shown in histograms are those of 200 bootstrapped inversion results. Top left panel (b) shows contour plot of number of bootstrapped solutions with specific values of moment and dip (with bins of $400 \times 10^{26}$ dyne.cm and $2^{\circ}$ ) for most common range of dip values.

low dip-slip sources are ill-conditioned, such that moment and dip are difficult to resolve independently (e.g. Kanamori and Given, 1981).

The rCMT method uses data with a similar period range as the W-phase moment tensor (Hayes et al., 2009), also in use at the NEIC, but its input signal is dominated by the surface waves, which arrive later and are of higher amplitude. Therefore, the rCMT requires a longer time window, but can also determine CMTs for large aftershocks, when the body wave signal (and thus the W-phase) may still be buried by the surface waves from a previous large event, such as some of the large aftershocks in the first day after the Tohoku mainshock. These methods also differ in the use of an inversion for centroid location (in the case of rCMT) as compared to the $\mathrm{W}$-phase grid search approach.

\section{2. rCMT Results for the Tohoku Earthquake}

When the initial event message was received from the USGS Earthquake Notification Service, indicating a magnitude 7.9 earthquake had occurred in the subduction zone offshore of Japan, a quick manual analysis was performed using the waveforms from only 5 nearby stations (see Fig. 1(a)). The initial results were determined within 23 minutes of the origin time of the event and showed a reverse faulting mechanism, with a shallow depth of $24 \mathrm{~km}$ and a moment magnitude of 8.9. NEIC staff was immediately informed of the significant increase in magnitude relative to its initial value. The fully automatic rCMT analysis was sent out to its mailing list 10 minutes later, and showed a very similar result (Fig. 1(b)), based on the data from 21 waveforms from 7 stations. A final solution was determined when all waveform data were available and is 
shown in Fig. 1(c). The moment magnitude for the final rCMT solution, at $M_{\mathrm{w}} 9.0$, is slightly higher than the initial results, but is slightly smaller than the gCMT (Fig. 1(d)), which has a moment magnitude of 9.1. Due to the bandpass of the used input signal, it may be that the total very long period energy content of the event is somewhat underestimated. Other factors that could lead to a different moment estimate than the gCMT solution are the use of a different global velocity model and the trade-off between moment and dip (see below). However, it is important to note that within 23 minutes of the earthquake a moment magnitude was determined that was within 0.2 magnitude unit of the "final", gCMT, value, a great improvement over the timeline of the results for the 2004 great Sumatra earthquake.

The rCMT system also carries out a bootstrapping analysis, using 200 inversions with stations resampled with replacement from the original input list. Histograms and $P / T / N$ axes of the focal mechanisms show the distribution of the source parameters from the 200 solutions (see Fig. 2, Polet et al., 2008). These automatic bootstrapping results are currently also made available through Twitter (from@CPPGeophysics). Our goal with this bootstrapping analysis is to produce meaningful error parameters for near real-time rCMT solutions, to help in the decision making process after the occurrence of a large event and also to provide uncertainty estimates for derived products, such as predicted tsunami waveheights. Additionally, the bootstrapping results may eventually be used to incorporate a priori knowledge of the fault plane orientation (in particular dip) in providing better constraints on the moment magnitude for shallow earthquakes. This could be achieved by leveraging the bootstrapping data as shown in Fig. 2(b), a contour plot of number of bootstrapped solutions with specific values of moment and dip (with bins of $400 \times 10^{26}$ dyne.cm and $2^{\circ}$ ), illustrating the dip/moment trade-off issue previously mentioned. However, the overall bootstrapping results for the Tohoku earthquake showed that the rCMT solution had a well constrained mechanism, depth and moment magnitude, the latter being particularly important given the 1.0 magnitude increase compared to the preliminary value.

\section{Rapid Prediction of Offshore Waveheights}

We have developed a method for Probabilistic Tsunami Hazard Analysis (PTHA) (Thio et al., 2010) based on the summation of subfault tsunami Green's functions for subduction zones along the Pacific Rim. The library of Green's functions that was developed for this purpose also enables us to quickly compute offshore waveheights for earthquake scenarios, including the recent Tohoku event.

The underlying principle for this approach is the linear behaviour of tsunami waves in deep water. This enables us to deconstruct a tsunami that is generated by an earthquake into a sum of individual tsunami waveforms (Green's functions) from a set of subfaults that adequately describes any significant earthquake rupture. By pre-computing and storing the tsunami waveforms at points along the coast, as generated by each subfault for a unit slip $(1 \mathrm{~m})$, we can efficiently synthesize tsunami waveforms for any slip distribution by summing the individual subfault tsunami waveforms (weighted by their slip). The same principle is used
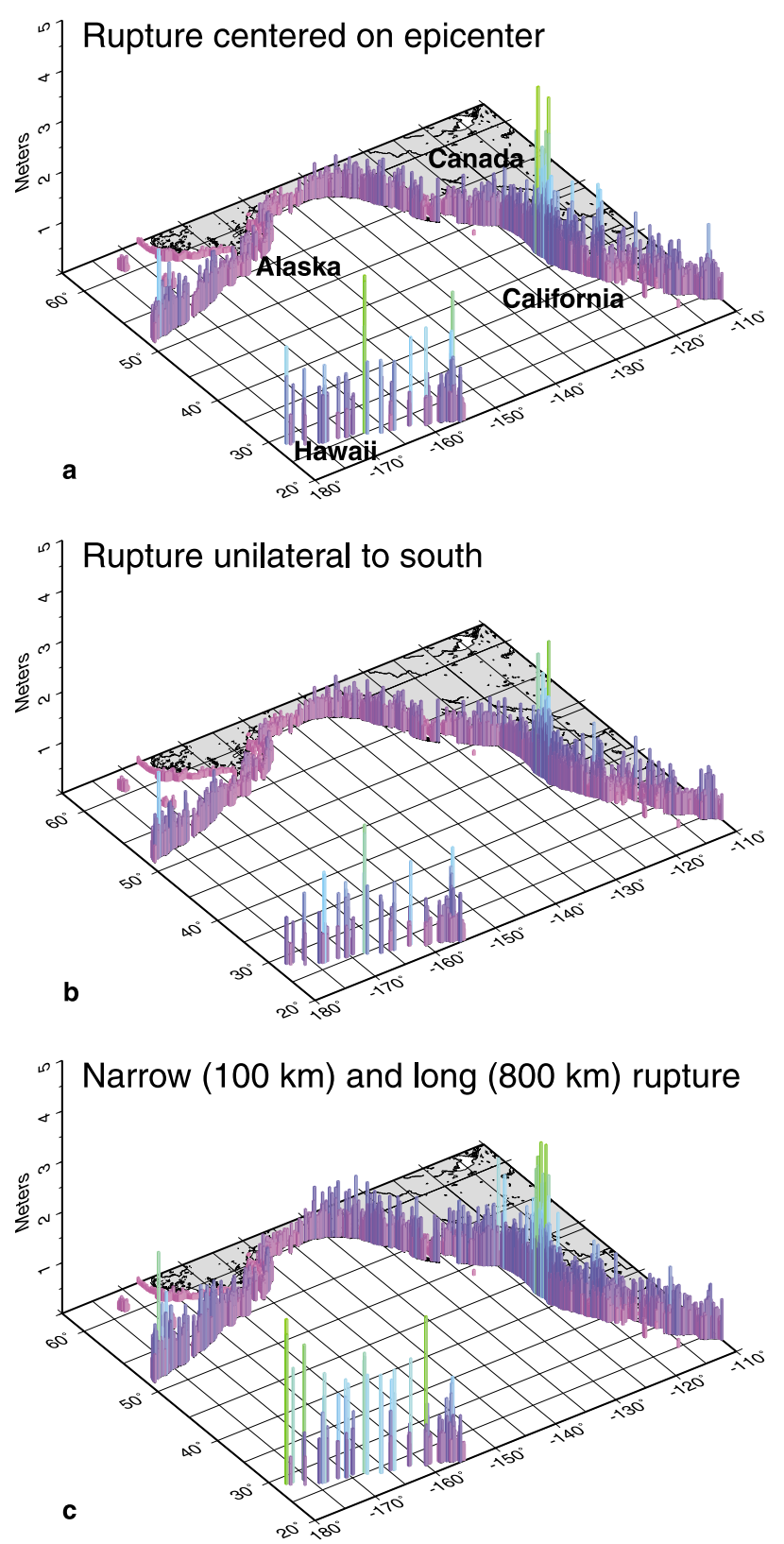

Fig. 3. Predicted offshore waveheights along the North America coastline for three different rupture models: a-uniform rupture centered on the hypocenter, $\mathrm{b}$ - uniform rupture extending south of the hypocenter, $\mathrm{c}$ - uniform rupture along a narrow and long rupture along the entire Japan trench.

in the inversion of tsunami waves for earthquake rupture (e.g. Satake, 1995) as well as the NOAA Short-term Inundation Forecast for Tsunamis (SIFT, Gica et al., 2008) system. The database of Green's functions was originally developed for PTHA studies, for which the desired location of the waveheights was offshore at depth of 5-30 m. These waveheights might therefore slightly under-predict the actual shoreline waveheights.

In Fig. 3 we present rapid predictions of tsunami waveheights offshore North America using our pre-computed library of tsunami Green's functions. The simulations are based on the aforementioned rCMT solution (Fig. 1(b)). We computed three earthquake scenarios that are consistent with this rCMT solution, using source scaling rela- 


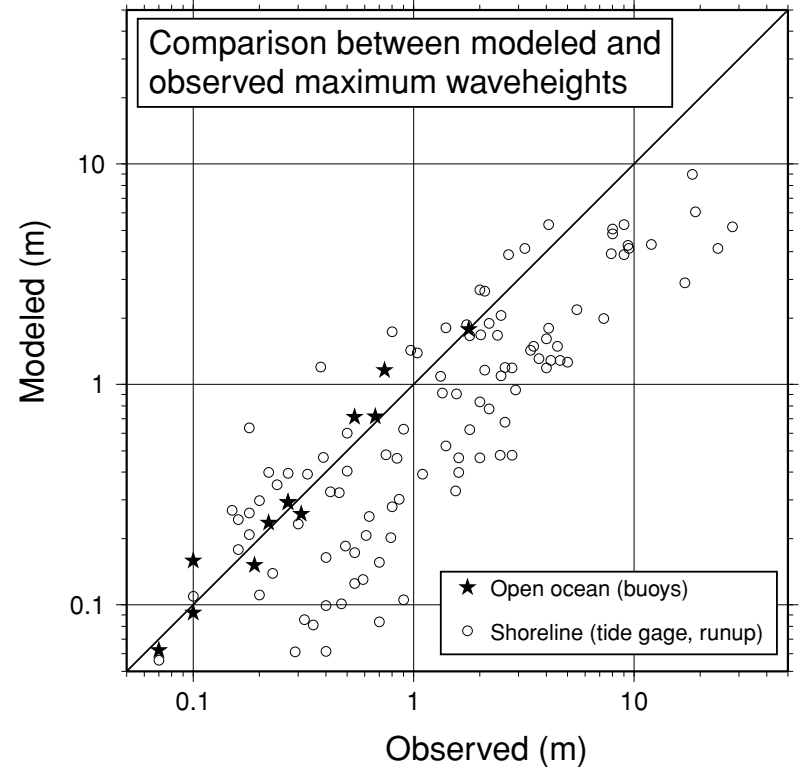

Fig. 4. Comparison between modeled and observed waveheights for the centered scenario (Fig. 1(b)). The solid stars are open ocean DART buoy measurements, open circles are shoreline and inundation waveheights.

tions for subduction zones (Papazachos et al., 2004). These scenarios are: a uniform rupture centered on the hypocenter, a uniform rupture extending south of the hypocenter, and a uniform rupture along a narrow and long rupture along the entire Japan trench. For the first two scenarios (Figs. 3(a) and 3(b)) the rupture length is $500 \mathrm{~km}$ and width is $150 \mathrm{~km}$. For the third (Fig. 3(c)), we used a narrower fault $(w=100 \mathrm{~km})$ and a length of $800 \mathrm{~km}$. In all cases, the slip is $8 \mathrm{~m}$, uniformly distributed along the entire rupture. The predicted waveheights are generally consistent with the observed waveheights and highlight the geographical variations observed along the California coast. For instance, in northern California, around Crescent City, and Southern Oregon, it predicts significantly larger waveheights (more than $2 \mathrm{~m}$ ) than both north and south of that region, which has been confirmed by observations. The comparison (Fig. 4) between observed and modeled waveheights shows that the open ocean observations are matched very well, and that the shoreline and inundation waveheights tend to be larger than the predicted offshore waveheights, as expected, but still correlate well. The differences are for a significant part due the to coarse grid size used for our Green's functions, which has the largest impact close to shore, and could be reduced by using a finer grid along the coast.

The efficiency of this method enables us to quickly generate a suite of tsunami waveheight predictions from possible scenarios that are consistent with the initial moment tensor solution, within minutes after receiving it. In this case, we sent out the waveheight predictions $31 / 2$ hours after of the origin time. Had the system been automated, or even streamlined, the waveheight predictions could have been sent within minutes of completion of the rCMT solution. Therefore, the rCMT timelines for the Tohoku earthquake illustrate the potential for determining tsunami height estimates within 30 minutes after large global earthquakes (al-

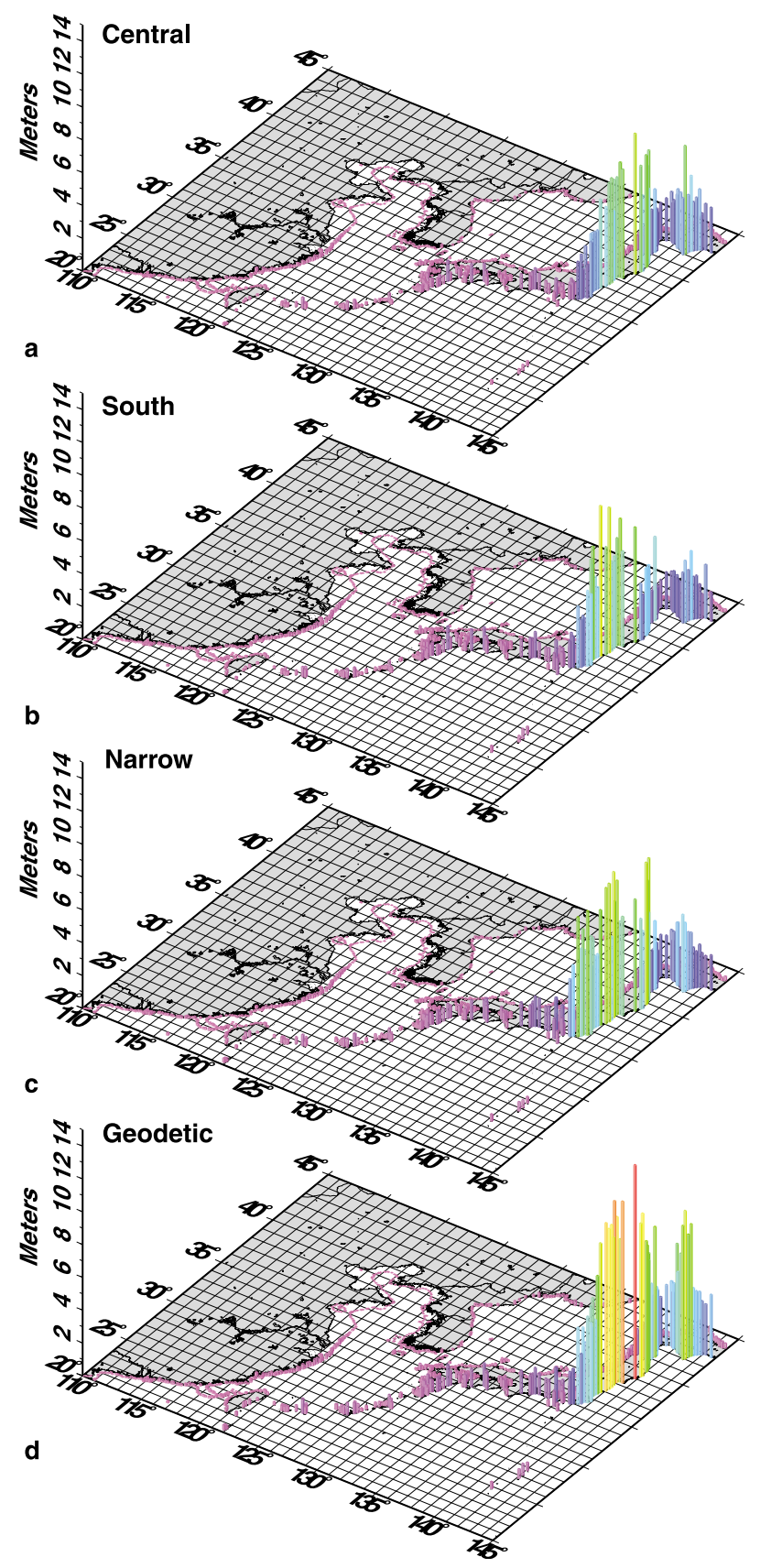

Fig. 5. Predicted offshore waveheights for Japan and China: a-c same models as in Fig. 3, d-preliminary model that has been derived from geodetic data (GSI, 2011).

though of course this time also depends on the GSN station coverage near the earthquake) and thus for regional tsunami warning.

In Fig. 5 we show a plot of predicted tsunami waveheights for Japan, Korea and northern China. The variability of waveheights for the three original scenarios is quite large in the near source region, but all show waveheights of more than 6-8 meters locally. For comparison, we also show the predicted waveheights (Fig. 5(d)) for the geodetic model, derived from GPS observations (GSI, 2011), which has high slip in the north $(30 \mathrm{~m})$ and less slip to the south along a narrower rupture. This model produces even higher tsunamis in the north. 


\section{Conclusion and Discussion}

The automated rCMT system provided an accurate estimate of the magnitude and mechanism of the 2011 Tohoku earthquake within 35 minutes of the earthquake origin time. By combining the automated rCMT procedure with tsunami Green's function summation techniques, we were able to rapidly predict tsunami waveheights along the Northern $\mathrm{Pa}-$ cific Rim, even though the tsunami calculation has not been automated yet. Due to the use of pre-computed Green's functions, the range of solutions that may be computed in a very short period of time is large, and a next step is to incorporate variability based on the rCMT bootstrapping results. We are also considering the use of a set of scenarios with heterogeneous slip, based on a systematic analysis of rupture models of previous megathrust earthquakes. These enhancements will provide a distribution of expected maximum waveheights, which can be updated and refined as more constraints on the rupture model or observed waveheight data become available.

Acknowledgments. We thank two anonymous reviewers and Gavin Hayes for their helpful suggestions to improve this manuscript. The rCMT part of this project was supported by the US Geological Survey. We would also like to thank Paul Earle at the NEIC for his help with rCMT.

\section{References}

Dziewonski, A. M., T. A. Chou, and J. H. Woodhouse, Determination of earthquake source parameters from waveform data for studies of global and regional seismicity, J. Geophys. Res., 86, 2825-2852, 1981.

Earle, P. S., D. J. Wald, K. S. Jaiswal, T. I. Allen, K. D. Marano, A. J. Hotovec, M. G. Hearne, and J. M. Fee, Prompt Assessment of Global
Earthquakes for Response (PAGER): A system for rapidly determining the impact of global earthquakes worldwide, U.S. Geological Survey Open-File Report, 2009-1131, 2009.

GSI (Geospatial Information Authority of Japan), Crustal Deformation and Fault Model obtained from GEONET data analysis (Preliminary), http://www.gsi.go.jp/cais/topic110313-index-e.html, 2011.

Gica, E., M. Spillane, V. V. Titov, C. Chamberlin, and J. C. Newman, Development of the forecast propagation database for NOAA's Shortterm Inundation Forecast for Tsunamis (SIFT), NOAA Tech. Memo. OAR PMEL-139, 89 pp, 2008.

Hayes, G. P., L. Rivera, and H. Kanamori, Source inversion of the Wphase; real-time implementation and extension to low magnitudes, Seismol. Res. Lett., 80, 817-822, 2009.

Kanamori, H. and J. W. Given, Use of long-period surface waves for rapid determination of earthquake source parameters, Phys. Earth Planet. Inter., 27, 8-31, 1981.

Kawakatsu, H., Centroid single force inversion of seismic waves generated by landslides, J. Geophys. Res., 94, 12363-12374, 1989.

Papazachos, B. C., E. M. Scordilis, D. G. Pangiatopoulos, C. B. Pazazachos, and G. F. Karakaisis, Global relations between seismic fault parameters and moment magnitude of earthquakes, Bull. Geol. Soc. Greece, XXXVI, 1482-1489, 2004.

Polet, J. and H. Kanamori, Automated CMT inversion using long period surface waves, Eos Trans. AGU, 76, Fall Meet. Suppl., 1995.

Polet, J., H. K. Thio, and P. Earle, Implementation of near real-time methods using surface waves to determine earthquake source characteristics at the National Earthquake Information Center, Eos Trans. AGU, 89(53), Fall Meet. Suppl., Abstract S13B-1801, 2008.

Satake, K., Linear and nonlinear computations of the 1992 Nicaragua earthquake tsunami, Pure Appl. Geophys., 144, 455-470, 1995.

Thio, H. K., P. G. Somerville, and J. Polet, Probabilistic tsunami hazard in California, Pacific Earthquake Engineering Research Center Report, 2010/108, 331 pp, 2010.

Wald, D. J., L. Wald, B. Worden, and J. Goltz, ShakeMap-A tool for earthquake response, U.S. Geological Survey Fact Sheet, 087-03, 2003.

J. Polet and H. K. Thio (e-mail: hong_kie_thio@urscorp.com) 